\title{
Immunophenotypic Markers Associated with Minimal Residual Disease Status and Outcome in Patients with Multiple Myeloma Undergoing Autologous Stem Cell Transplantation
}

\author{
Missassi G', Ikoma-Colturato MRV $^{2 *}$, Simioni AJ $^{3}$, \\ Bortolucci CM², Conte-Spilari J E ${ }^{2}$, Souza $\mathrm{MP}^{3}$ and \\ Colturato VAR $^{3}$ \\ ${ }^{1}$ Multiprofessional Residency in Cancer Attention, \\ Hospital Amaral Carvalho, J aú, São Paulo, Brazil \\ ${ }^{2}$ Flow Cytometry Laboratory, Hospital Amaral Carvalho, \\ Jaú, São Paulo, Brazil \\ ${ }^{3}$ Bone Marrow Transplantation Service, Hospital Amaral \\ Carvalho, J aú, São Paulo, Brazil \\ *Corresponding author: Ikoma-Colturato MRV, Flow \\ Cytometry Laboratory, Hospital Amaral Carvalho, Dona \\ Silveria Street, 150, J aú, Zip Code: 17210-080, São Paulo, \\ Brazil
}

Received: April 26, 2021; Accepted: May 26, 2021; Published: June 02, 2021

\begin{abstract}
Multiple Myeloma (MM) is one of the most common hematologic malignancies, with a heterogeneous prognosis. Therefore, the recognition of biomarkers can be useful to understand the differences in patient outcomes. Minimal Residual Disease (MRD) has been considered a very important prognostic factor in MM. In parallel, the prognostic value of immunophenotypic markers expressed in MM Plasma Cells (PCs) has also been described. The aim of this study was to assess the impact of CD27, CD28, CD45, CD56, CD117 and $\beta 2$-microglobulin expressions on the outcome of $154 \mathrm{MM}$ patients undergoing Autologous Stem Cell Transplantation (ASCT). The relation of each marker studied with the Overall Survival (OS) and Progression-Free Survival (PFS) was assessed, alone and in association with pre-ASCT MRD. Scores of good (GPM) and poor Prognostic Markers (PPM) were established, according to their respective survival curves. The expressions of CD27 and CD45 were associated to longer OS ( $p=0.013$ and $p=0.00$, respectively) and PFS ( $p=0.00)$ as well as the absence of CD28 (OS $p=0.026$; PFS $p=0.001$ ) and CD56 (OS $p=0.004$; PFS $p=0.009$ ), in patients with undetectable MRD. The number of GPM showed an inverse correlation with the level of MRD $(p=0.04)$, while a higher number of PPM was observed in patients with higher levels of MRD $(p=0.04)$, which were also significantly associated with OS and PFS.
\end{abstract}

In conclusion, although pre-ASCT MRD is a powerful prognostic factor in $\mathrm{MM}$, these biomarkers can provide additional prognostic information and be used in the follow-up of MM patients.

Keywords: Multiple myeloma; Minimal residual disease; Prognostic markers; Immunophenotype markers; Autologous stem cell transplantation

\section{Introduction}

Multiple Myeloma is the third most prevalent hematological malignancy. In the last twenty-six years, the incidence of MM has increased, as well as deaths due to the disease $[1,2]$. MM is a characterized by the expansion of clonal PCs in bone marrow, that can compromise the normal hematopoiesis and systemically affect the patients causing symptoms secondary to elevated calcium levels, renal failure, anemia and osteolytic bone lesions (CRAB) $[3,4]$.

Upfront consolidation with ASCT is one of the most effective therapies for $\mathrm{MM}$ in eligible patients, after the first line of chemotherapy [3-5]. ASCT has proven to increase OS and PFS in MM patients [6,7]. However, the majority of MM patients can relapse, despite achievement of Complete Remission (CR) $[5,8]$.

New therapeutic strategies are being introduced and biomarkers have received special attention to identify groups of patients with different prognostic factors, including the cytogenetic classification for risk stratification in MM [4,5,9-15]. Furthermore, MRD has proven to be one of the main predictive factor for relapse in $\mathrm{MM}$ patients eligible or not to ASCT [8,13,15-19]. A significant increase in PFS and OS was observed in patients who had undetectable MRD before and after ASCT $[13,15]$. On the other hand, persistent MRD resulted in reduced PFS and OS after ASCT $[15,17,20]$. Nowadays, Multiparametric Flow Cytometry (MFC) is one of the most sensitive methods recommended for monitoring MRD to assess efficacy of the treatment [21].

The immunophenotypic profile of clonal PCs is another prognostic parameter that has been investigated in MM patients [9-12]. Antigen expressions on clonal PCs varies according to the stage and biological characteristics of the disease [22]. Considering that more than $90 \%$ of plasma MM cells express an aberrant immunophenotypic profile $[12,23]$, the recognition of these specific surface antigens is useful for the evaluation of MRD and can provide additional information on the risk of relapse $[15,17,24]$.

The main immunophenotypic markers with prognostic implications, which were previously described in MM include CD27, CD28, CD45, CD56, CD117 and $\beta 2$-microglobulin [12,22,25].

CD27, a transmembrane phosphoglycoprotein member of tumor necrosis factor (TNF) family is expressed in normal plasma cells
Ann Hematol Oncol - Volume 8 Issue 8 - 2021 ISSN : 2375-7965 | www.austinpublishing group.com Ikoma-Colturato et al. (C) All rights are reserved
Citation: Missassi G, Ikoma-Colturato MRV, Simioni AJ, Bortolucci CM, Conte-Spilari JE, Souza MP, et al. Immunophenotypic Markers Associated with Minimal Residual Disease Status and Outcome in Patients with Multiple Myeloma Undergoing Autologous Stem Cell Transplantation. Ann Hematol Oncol. 2021; 8(8): 1357. 
[26,27]. This marker is frequently downregulated in MM plasma cells [28]. Its expression is involved in PCs differentiation [25] and is associated with higher survival rates $[28,29]$. On the other hand its absence leads to disease progression $[30,31]$.

CD28 is a disulfide-linked homodimer type I glycoprotein, member of the immunoglobulin superfamily, expressed on most $\mathrm{T}$ lineage cells, NK cell subsets, and neoplastic plasma cells [32,33]. This marker is a mediator of MM cell survival and chemotherapy resistance [32] and its expression is related with a more aggressive phenotype [27], cytogenetic abnormalities and lower OS and PFS [9].

CD45 is an important regulator of immune cells signaling [34] and it is associated with decreased bone marrow angiogenesis [35]. CD45 expression is also associated with longer OS in MM patients treated with high dose therapy [36]. Regarding CD56, also known as Neural Cell Adhesion Molecule (NCAM), controversial data about its prognostic relevance have been reported. The lack of CD56 on malignant plasma cells has been associated with worse prognosis and a higher incidence of extramedullary disease [37]. While Mateo et al (2008) did not observe a significant influence of CD56 expression on survival [9], other studies reported that the lack of this marker was associated with the presence of chromosome translocation [11,14], associated to poor prognosis [38,39].

CD117 is a receptor involved in cell growth and its presence has been related to higher survival rates and low levels of creatinine, that is, lower risks of renal failure associated with MM activity, which is a factor of poor prognosis $[9,40,41]$.

The other marker already described was $\beta 2$-microglobulin, whose intensity of expression on the surface of PCs has been associated to greater clinical outcomes of MM patients [42].

Considering the biological diversity of MM, more effective individualized therapies are necessary [43]. In order to identify patients with higher or lower risk of disease relapse, this study investigated the impact of immunophenotypic markers on MM patient survival, including their association with pre-ASCT MRD results.

\section{Materials and Methods}

\section{Ethics statement}

The study was approved by the institutional research ethics committee (process number: CAAE 17569119.0.0000.5434).

\section{Patients and study design}

This retrospective study used the databases of both the flow cytometry laboratory and the bone marrow transplantation service. 154 MM patients who underwent ASCT from June of 2013 to September of 2018 were included in this cohort. Fifty-seven patients (37\%) were female and ninety-seven (63\%) were male, aged 29 to 73 years. All patients had been previously treated with at least one chemotherapy regimen prior to ASCT. All of them had available the immunophenotypic markers and MRD data before transplantation. The median follow-up was 14 months.

Patients were separated into three groups according to MRD levels $(<0.01 \%, 0.01 \%$ to $1 \%$ and $>1 \%)$. During the cohort time, the levels of sensitivity of MRD tests varied from $10^{-4}(0.01 \%)$ by conventional
MFC to $10^{-6}(0.0001 \%)$ by Next Generation Flow (NGF). A cut-off of $<0.01 \%$ was used to consider MRD as "negative" or indetectable.

The impact of the expression of each marker on diagnosis, such as CD27, CD28, CD45, CD56, CD117 and $\beta 2$-microglobulin, was individually evaluated regarding the OS and PFS. According to the significant difference in survival curves, these markers were recognized as good (GPM) or Poor Prognostic Markers (PPM).

For each marker, one point was added to the score of either good or poor prognosis. The number of GPM and PPM were scored ranging from 0 to 5 for each patient. Patients were classified according to the number of GPM and PPM ( $\leq 2$ and $>3)$. The relation of these scores with survival rates were also assessed.

Two groups were defined considering the expression of $\beta 2$ microglobulin, in order to evaluate its prognostic value, according to previously described [42]: group 1 included patients with low or no expression of $\beta 2$-microglobulin, and group 2 with high expression of this marker.

\section{Flow cytometry}

Bone marrow samples collected in EDTAK2 tubes were processed within 24 hours of collection. Euroflow standard operating procedures were used for instrument setup, sample preparation, data acquisition and analysis as previously described [44]. For the diagnosis of MM, the samples were stained with an 8-color combination of monoclonal antibodies (MoAb) in 2 tubes as previously described [45]: (1) CD45HV450 (2D1), CD138PO(B-A38), CD38FITC(HB7), CD56PE (MY31), $\quad \beta 2$-microglobulin PerCPCy5.5 (TÜ99), CD19PECy7 (J3119), cytoplasmic (cy)Kappa APC (C022), cyLambda APCH7(1155-2), and (2) CD45HV450(2D1), CD138PO (B-A38), CD38 FITC (HB7), CD28PE (L293), CD27PerCPCy5.5 (L128), CD19PECy7 (J3119), CD117 APC (104D2), CD81APCH7 (JS-81). Pre-ASCT MRDs were evaluated using the conventional 8-color MFC, from June 2013 to September 2014. Next Generation Flow (NGF) were the methodology used from that date. The samples used to evaluate NGF-MRD were prepared using bulk-lysis prior to staining with MoAb, to allow recovery of $>107$ cell events in most samples [46]. 8-color MM MRD combinations included 2 tubes: CD19PECy7 (J3119), CD27BV510 (O323), CD38FITC (HB7 or CYT-38F2), CD56PE (MY31), CD45PerCPCy5.5 (HI30), CD138BV421 (MI15) in both tubes, used as backbone markers [46]. They were combined with cyKappa APC (C022) and cyLambda APCH7 (1-155-2) in the first tube and CD117APC (104D2) and CD81APCH7 (JS-81) in the second tube, according to Euroflow recommendations $[44,46]$. A minimum of $1 \times 10^{6}$ cell events per tube was acquired for diagnostic samples and for conventional MFC-MRD samples, while at least $5 \times 10^{6}$ events per tube were acquired for NGF-MRD assessments, using a BD FACSCanto II 8-color flow cytometer/3lasers (BD Biosciences, San Jose, CA) and the FACSDiva software (BD Bioscience). The MRD detection and quantification limits were $10^{-4} / 10^{-5}$ for conventional MFC and $10^{-6}$ for NGF-MRD. Infinicyt ${ }^{\mathrm{TM}}$ software (Cytognos SL, Salamanca, Spain) was used for MFC data analysis.

\section{Statistical analysis}

PFS was defined as the time from ASCT to progression or death from any cause and OS as the time from ASCT to death. Kaplan-Meier method and the two-sided log-rank test for each group were used to 

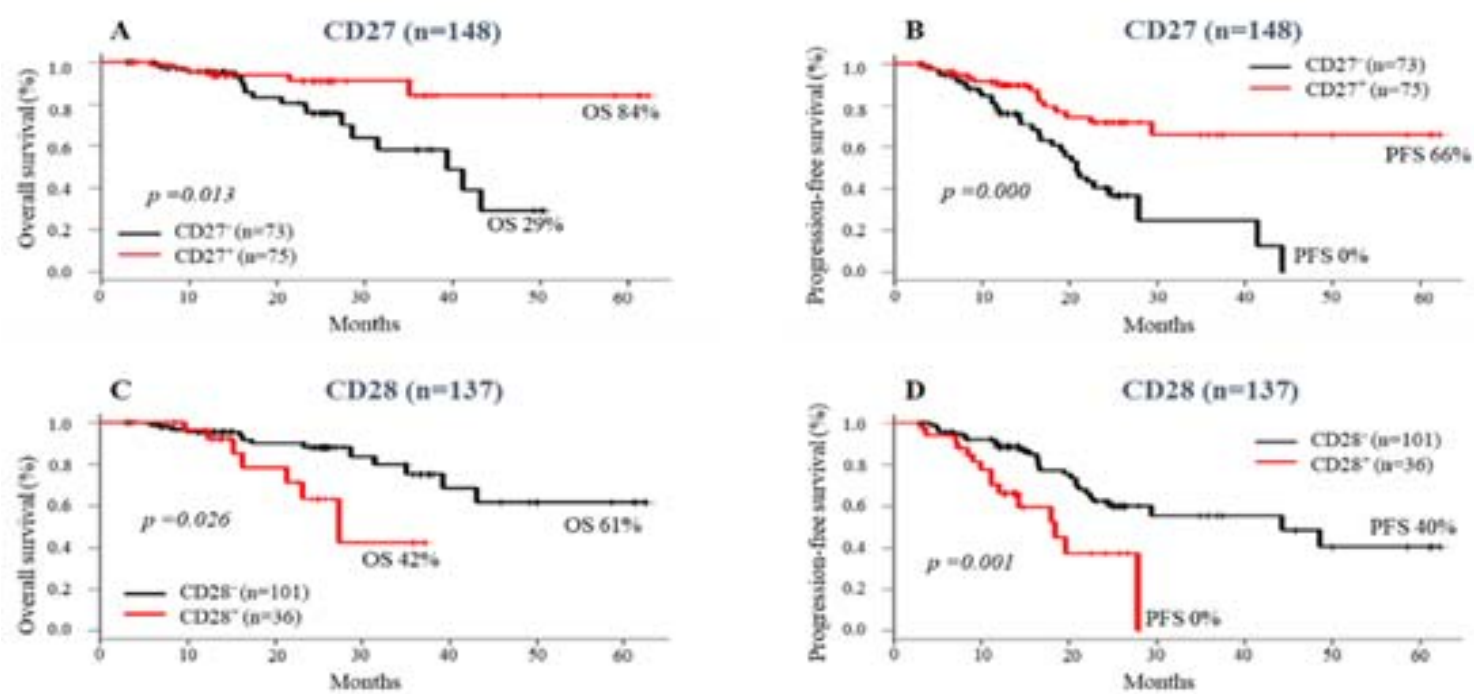

E.

CD45 ( $\mathrm{n}=153)$
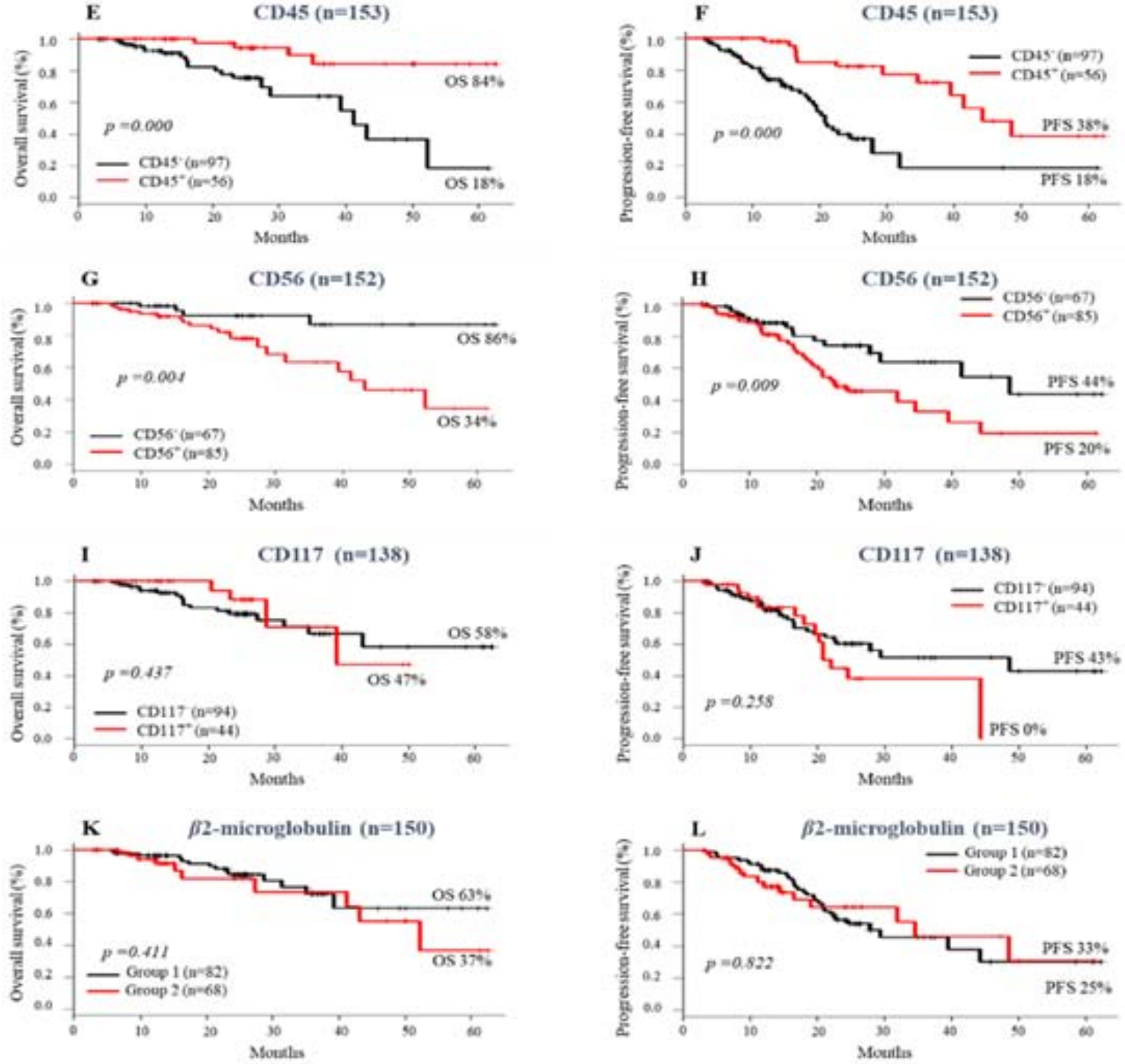

Figure 1: Impact of Immunophenotype makers on survival of MM patient's pre ASCT. Overall survival and Progression-free survival according to the presence or absence of Immunophenotype markers. 


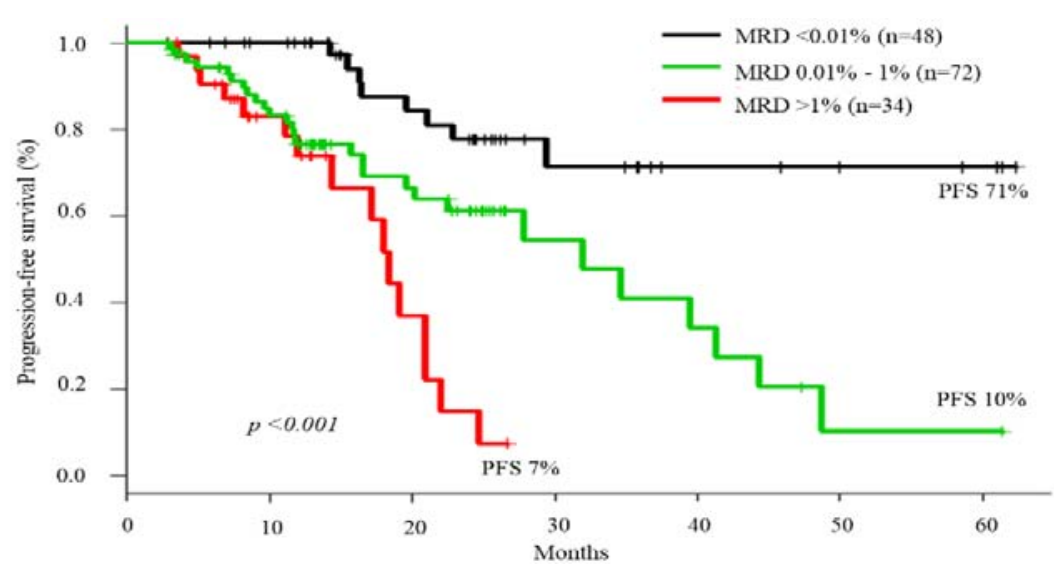

Figure 2: Progression- free survival of Multiple Myeloma patients according to pre ASCT MRD level.

plot and compare OS and PFS. A Cox proportional hazard regression was used to evaluate the prognostic value of immunophenotypic markers. Pearson's coefficient was used to calculate the correlations between MRD and the number of GPM and PPM. The tests were performed in the R 3.4.2 [47] environment software, with a statistically significant predictive value of $\mathrm{p}<0.05$.

\section{Results}

\section{Prognostic value of immunophenotypic markers}

The presence or absence of most immunophenotypic markers had an impact on patient survival. The isolated expressions of CD27 and CD45 were significantly related to higher OS ( $84 \%$ vs $29 \%, \mathrm{p}=$ 0.013 for CD27; $84 \%$ vs $18 \%, \mathrm{p}=0.00$ for CD 45$)$ and PFS (66\% vs $0 \%$ for $\mathrm{CD} 27 ; 38 \%$ vs $18 \%$ for $\mathrm{CD} 45 ; \mathrm{p}=0.00$ for both) when compared with cases without expressions of these markers (Figure 1A-B and E-F). On the other hand, the expressions of CD28 and CD56 were significantly associated to reduced survival rates: OS $=42 \%$ vs $61 \%$ $(\mathrm{p}=0.026)$ and $\mathrm{PFS}=0 \%$ vs $40 \%(\mathrm{p}=0.001)$ for $\mathrm{CD} 28$; OS $=34 \%$ vs $86 \%$ $(\mathrm{p}=0.004)$ and PFS 20\% vs 44\% ( $\mathrm{p}=0.009)$ for CD56 (Figure 1C-D and $\mathrm{G}-\mathrm{H})$. No differences were noticed in OS and PFS regarding CD117 $(\mathrm{OS}, \mathrm{p}=0.43$; PFS, $\mathrm{p}=0.25$ ) and $\beta 2$-microglobulin (OS, $\mathrm{p}=0.41$; PFS, $\mathrm{p}=0.82$ ) expressions (Figure $1 \mathrm{I}-\mathrm{L}$ ). Although the high intensity of membrane $\beta 2$-microglobulin has been associated with better SG rates [43], our data did not show a prognostic value for this marker, since patients with different intensities of $\beta 2$-microglobulin expression had survival rates similar.

\section{Association of MRD status and immunophenotypic markers on survival of MM patients}

As expected, in this cohort, MRD levels $<0.01 \%$ ( $\mathrm{n}=48$ ) were associated with better clinical outcomes with significantly longer PFS $(\mathrm{p}<0.001)$ when compared to groups with MRD $>0.01 \%$ to $1 \%$ and $>1 \%$ (Figure 2).

In the group of patients with MRD $<0.01 \%$, the expressions of CD27 and CD45 in neoplastic PCs were associated with a significantly higher probability of OS ( $p=0.00$ for both) and PFS ( $p=0.00$ for both), when compared to the absences of CD27 and CD45 expressions (Figure $3 \mathrm{~A}-\mathrm{B}$ and E-F)

The opposite was observed in patients with negative MRD, whose neoplastic PCs expressed CD28 and CD56. They had a significantly lower probability of OS ( $p=0.023$ and $p=0.009$, respectively for $\mathrm{CD} 28$ and CD56), compared to cases without these markers (Figure 3C-D and $\mathrm{G}-\mathrm{H})$. However, the PFS was similar among groups $(\mathrm{p}=0.44$ and $p=0.46$, respectively). The presence or absence of CD117 and $\beta 2$ microglobulin expressions had no impact on survival curves of MM patients with negative MRD (Figure 3I- L).

On the other hand, in patients with MRD $>0.01 \%$, the expression of CD117 was associated to longer OS ( $46 \%$ vs 22\%, p=0.048). The absence of CD28 expression also demonstrated a statistical trend towards longer PFS ( $20 \%$ vs $0 \%, \mathrm{p}=0.07)$ when compared to patients with CD28 positive PCs. The other markers had no impact on the outcome of this group of patients (Figure 4).

\section{Score of immunophenotypic markers and correlation with MRD status}

As demonstrated above, the expressions of CD27, CD45 and CD117, as well as the lack of CD28 and CD56 were considered GPM. The expressions of CD28 and CD56 and the absence of expressions of CD27, CD45 and CD117 were considered PPM.

The results showed that higher numbers of GPM were associated with lower levels of MRD $(R=-0.197, p=0.04)$, while higher numbers of PPM were observed in patients with higher levels of MRD $(\mathrm{R}=$ $0.197, \mathrm{p}=0.04$; Figure 5A-B).

Analysis of the influence of the number of GPM and PPM on OS and PFS revealed that patients who had $>3$ GPM had longer OS ( $83 \%$ vs $31 \%, \mathrm{p}=0.0003)$ and PFS $(60 \%$ vs $14 \%, \mathrm{p}=0.00)$ compared to the group with $\leq 2$ GPM (Figure 6A-B). Patients with $\leq 2$ PPM had significant longer OS $(82 \%$ vs $31 \%, \mathrm{p}=0.001)$ and PFS $(60 \%$ vs $13 \%$, $\mathrm{p}=0.00$ ) than patients with $\geq 3$ PPM (Figure 6C-D).

However, the association of immunophenotypic prognostic markers and MRD status did not show any prognostic evidence, except in the group with $\leq 2 \mathrm{PPM}$ and negative MRD, which had a longer PFS compared to the group with $\geq 3$ PPM (79\% vs 40\%, p= 0.027) (Figure 7D). A trend towards higher rates of OS and PFS was observed in patients with $\geq 3 \mathrm{GPM}$, regardless of the status of negative (Figure 7A-B) or positive MRD (Figure 8A-B). 

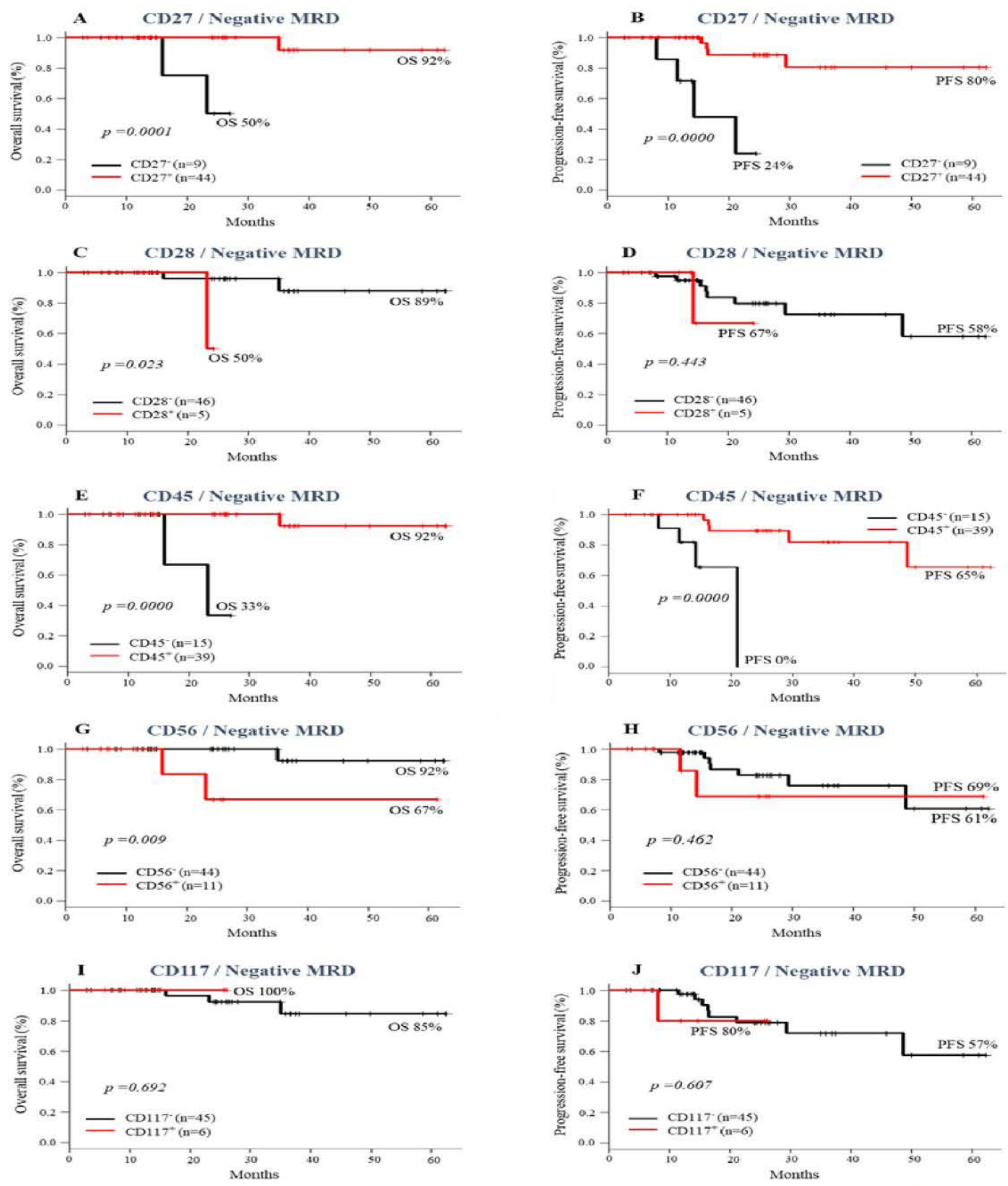

K B2-microglobulin/Negative MRD

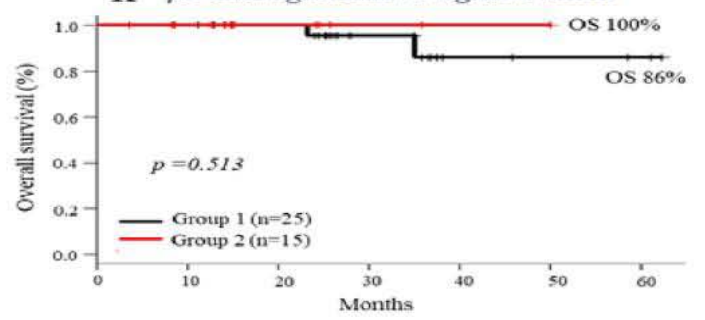

L $\beta 2$-microglobulin / Negative MRD

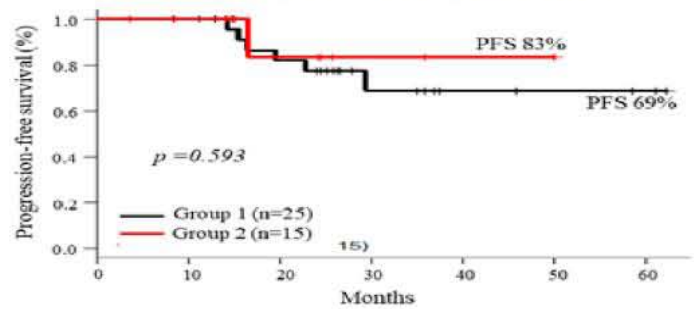

Figure 3: Impact of the Presence or absence of immunophenotypic markers and MRD $<0.01 \%$ pre ASCT on survival of MM patients. Overall survival and progression-free survival. 

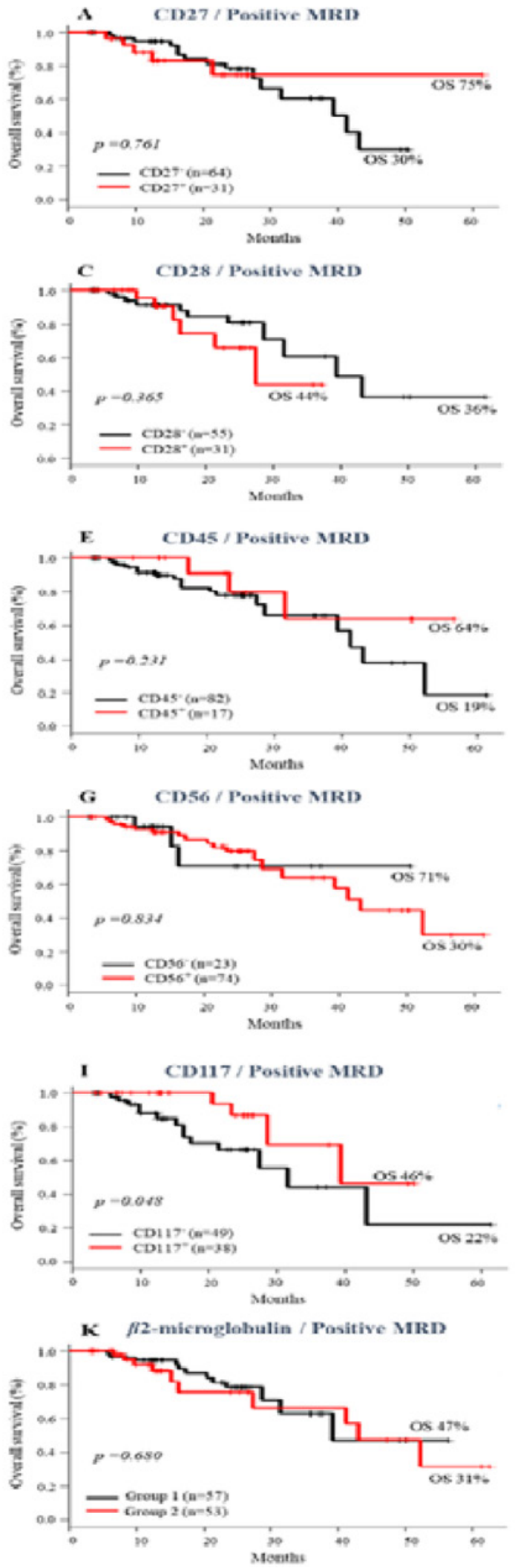
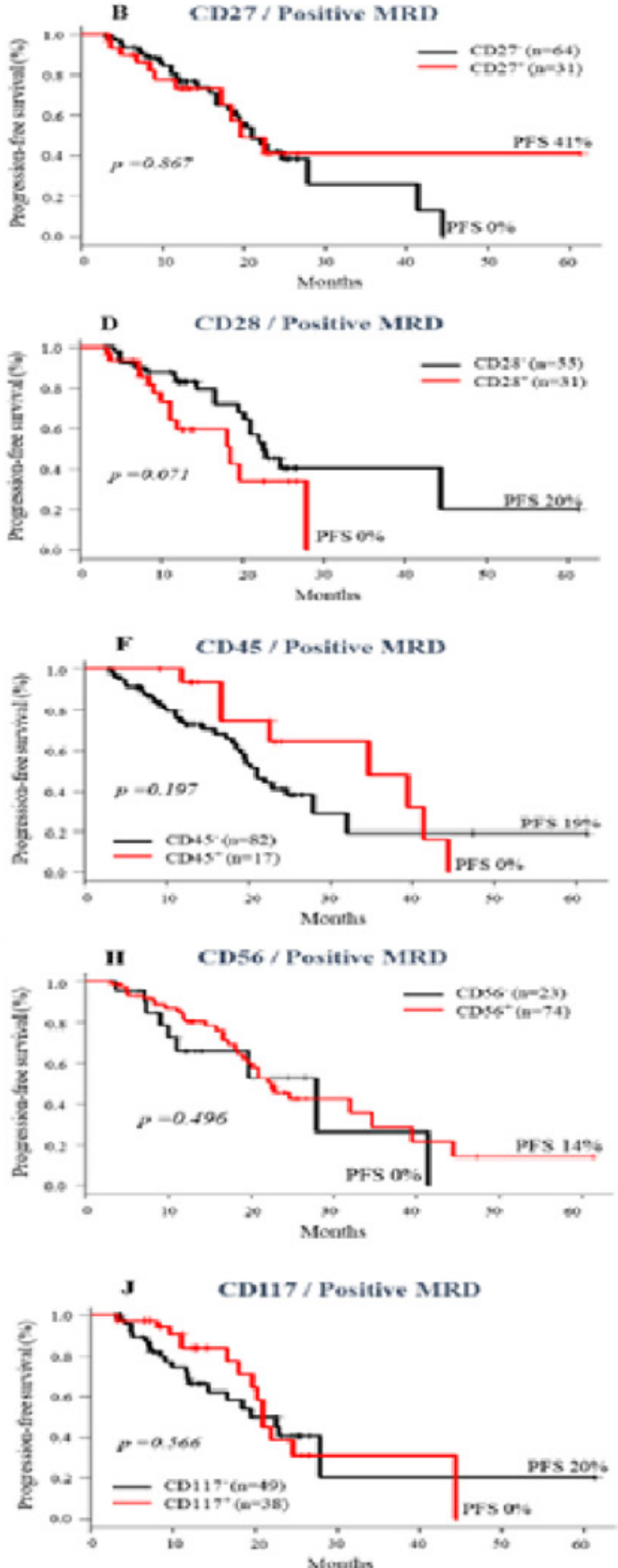

L $/$ 2-microglobulin / Positive MRD

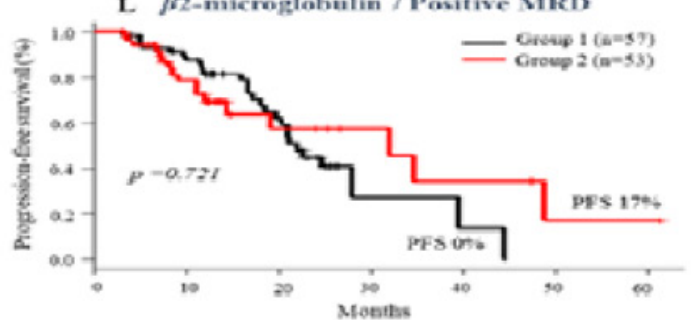

Figure 4: Impact of the Presence or absence of immunophenotypic markers and MRD $<0.01 \%$ pre ASCT on survival of MM patients. Overall survival and progression-free survival. 

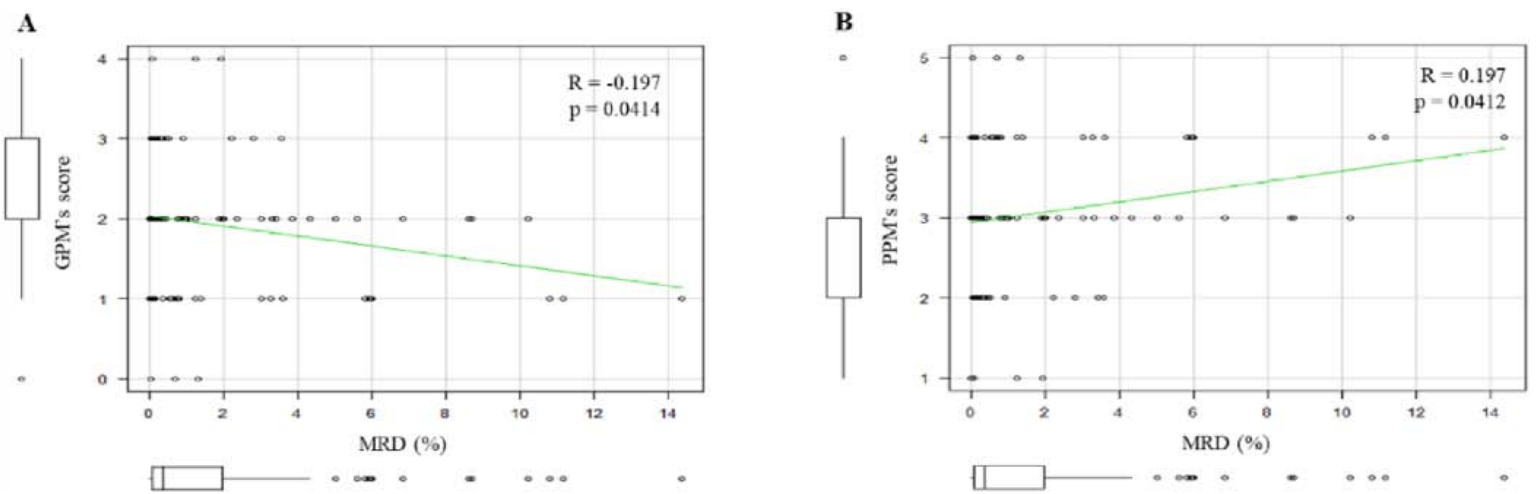

Figure 5: Correlation between scores of immunophenotypic markers and pre-ASCT MRD levels in patients with MM. (A) Good Prognostic Markers (GPM) and (B) Poor Prognostic Markers (PPM).
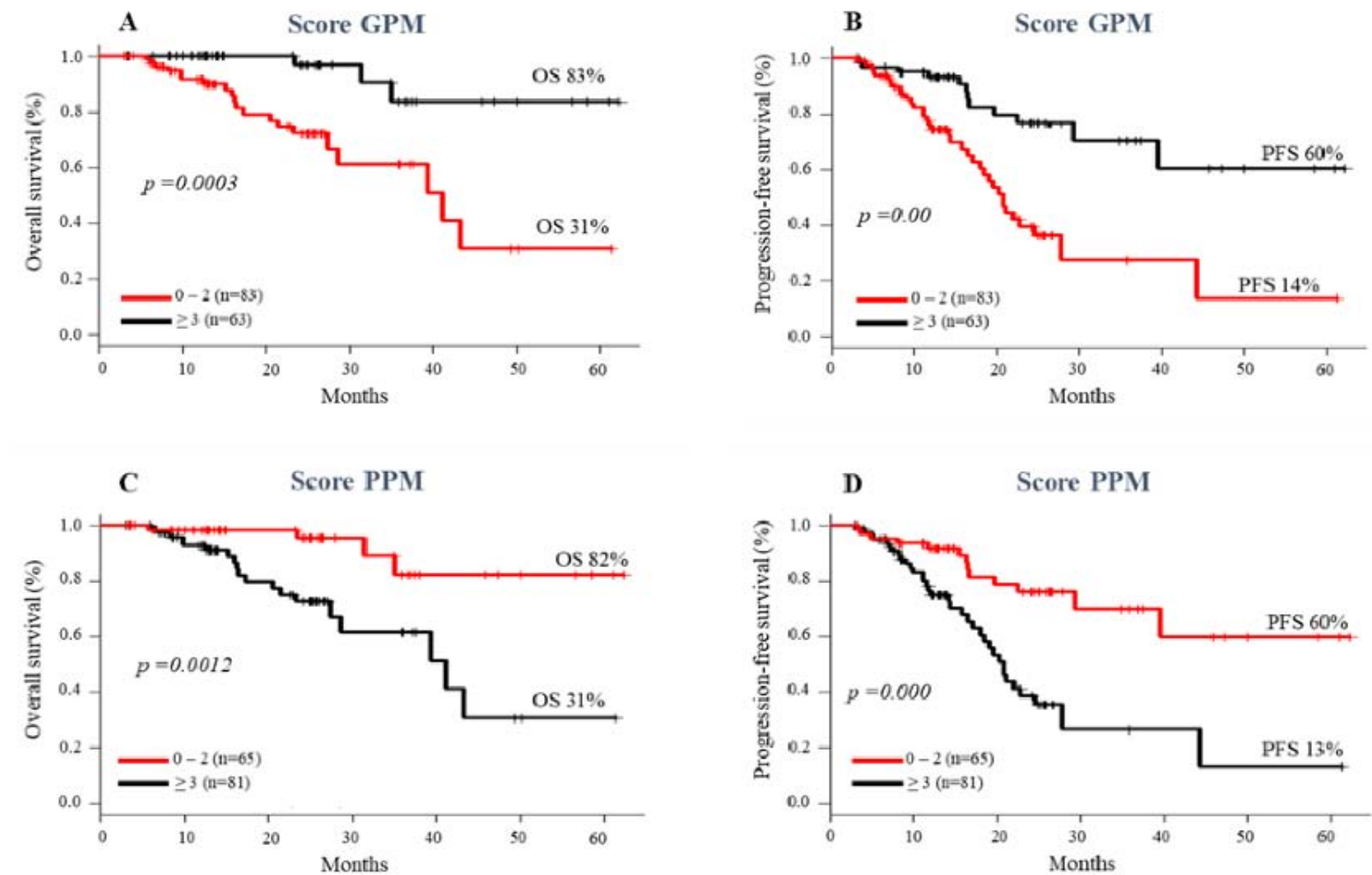

Figure 6: Impact of scores of Good Prognostic Markers (GPM) and Poor Prognostic Markers (PPM) on survival of MM patients. Overall survival (A and C) progression-free survival (B and $D)$.

\section{Discussion}

Unlike other studies, we compared not only the impact of immunophenotypic markers on survival, but also their association with MRD results on the outcome of MM patients.

Although prognostic marker expressions have been correlated with the survival of MM patients, the differences in study design and in the methodologies used in previous reports have prevented the reaching of a consensus on their role $[9,22]$.

Our results reinforced the impact of pre-ASCT MRD as an important prognostic factor in MM patients $[15,20,48]$. Undetectable
MRD was associated with higher probability of PFS [13,16,49]. Additionally, CD27, CD28, CD45 and CD56 are immunophenotypic markers which individually also demonstrated an impact on survival of MM patients.

The absence of CD27 expression in MM plasma cells has been associated with disease relapse, while its expression was associated with increased OS [28]. Indeed CD27 expression was described to be significantly higher in patients who achieved complete remission when compared with newly diagnosed and relapsed patients [31]. In our series of patients, CD27 expression associated with MRD negativity before ASCT conferred a better clinical outcome. The same 
Ikoma-Colturato MRV

Austin Publishing Group

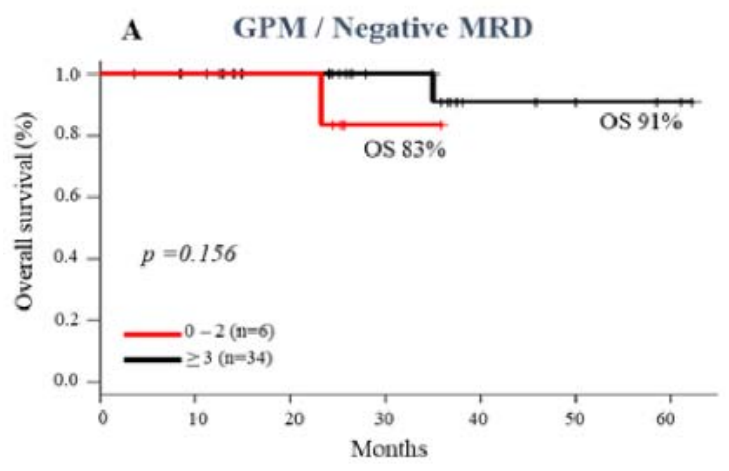

C PPM/ Negative MRD

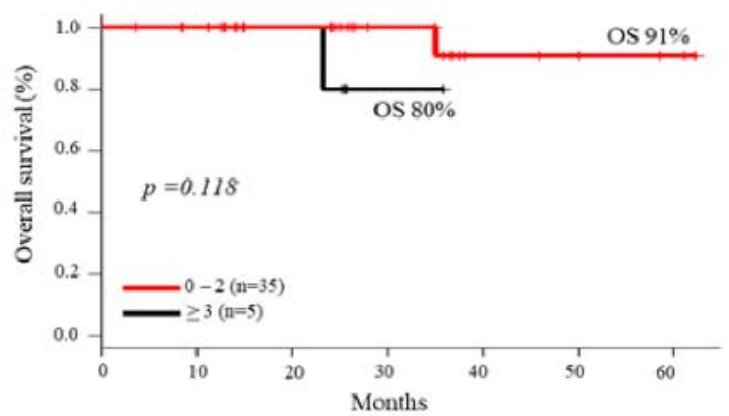

B

GPM / Negative MRD

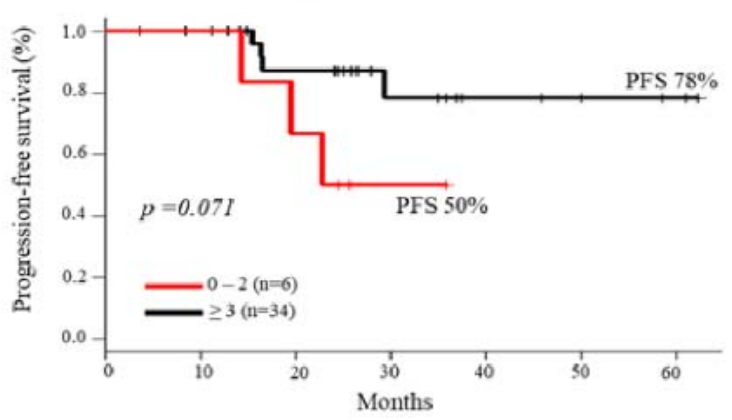

D PPM/ Negative MRD

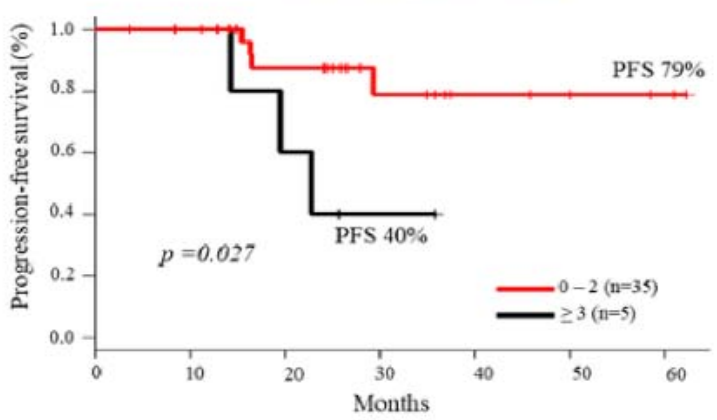

Figure 7: Impact of scores of Good Prognostic Markers (GPM) and Poor Prognostic Markers (PPM) on survival of MM patients with negative MRD pres ASCT. Overall survival ( $A$ and $C)$; progression-free survival (B and $D)$.

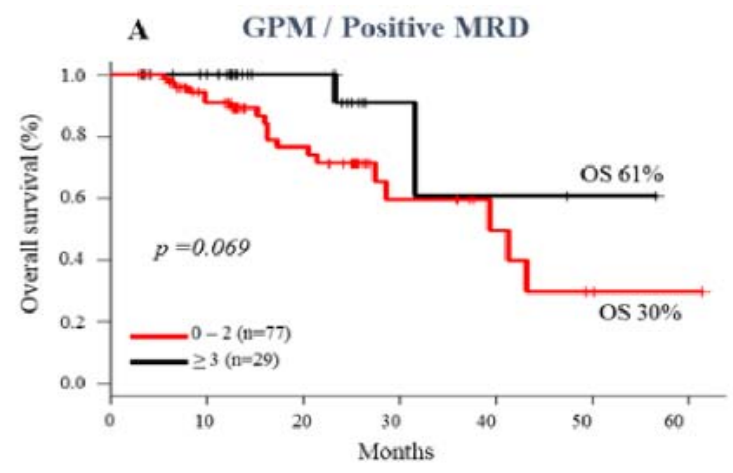

C PPM / Positive MRD

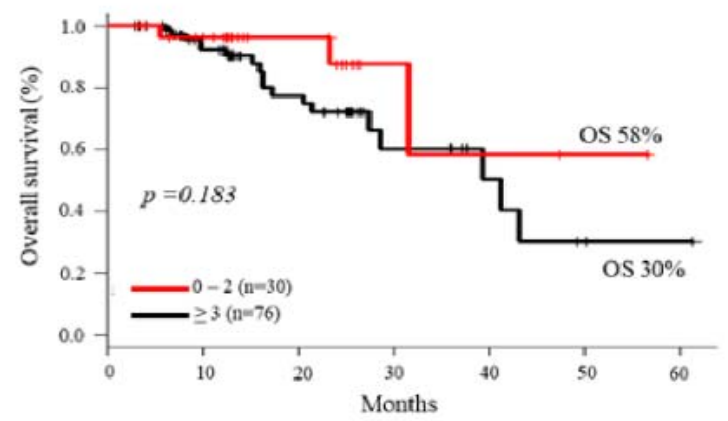

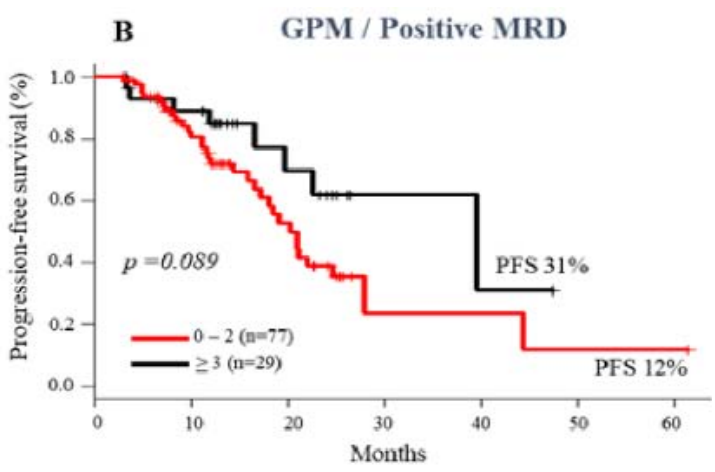

D PPM / Positive MRD

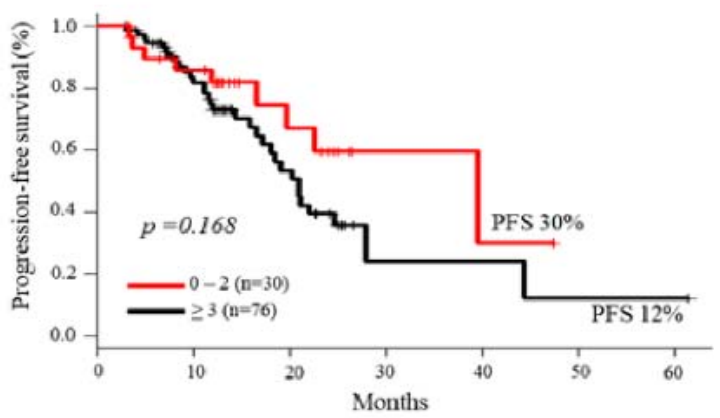

Figure 8: Impact of scores of Good Prognostic Markers (GPM) and Poor Prognostic Markers (PPM) on survival of MM patients with positive MRD pres ASCT. Overall survival ( $A$ and $C$ ); progression-free survival (B and $D)$.

Submit your Manuscript | www.austinpublishing group.com

Ann Hematol Oncol 8(8): id1357 (2021) - Page - 08 
was observed with CD45 expression.

CD45 is expressed mainly in early stages of PC maturation and its expression decreases throughout this process [50]. In monoclonal plasma cells, the absence of this marker has been related to resistance to apoptosis and continuous proliferation, which favors disease progression [35,51]. This may explain the higher survival rates of patients with MM who have CD45 expression on their clonal PCs observed in this study.

Therefore, we propose that the expressions of CD27 and CD45, in association with a condition of undetectable MRD, can identify a subgroup of MM patients with very good prognosis risk.

Another surface marker analyzed was CD56, an adhesion molecule involved in anchoring PCs in the bone marrow stroma. This has been the explanation for the leukemic phase of MM being more frequent in patients without CD56 expression in PCs [52]. The absence of CD56 in MM plasma cells was associated with poor prognosis, more aggressive disease [37] and lower PFS [11,19,38] and OS [41,53], however this association was not consensual [9]. Our study showed that the absence of CD56 in clonal PC was associated to significantly higher probability of OS and PFS post ASCT. Also, patients with negative MRD and who lack CD56 at the time of diagnosis had a longer OS, however this did not occur in PFS. It should be emphasized that the differences in the methods used for CD56 detection (immunohistochemistry and MFC) can lead to differences in results between previous studies $[40,52,54]$. This must be taken into account when comparing these results. Another factor that could influence the results was the low number of plasma cells detected by conventional MFC, even in diagnostic samples. The acquisition of a large number of cells is essential for an adequate analysis of abnormal subclones of PC [46].

The poor prognosis associated to CD28 is probably because it is one of the main molecules responsible for the survival and proliferation of clonal PCs [32,33]. Corroborating this, the absence of CD28 was associated with an increased probability of survival in our series. We observed higher rates of OS in patients with both CD28 and MRD negative pre-ASCT.

Although the absence of CD117 expression has been considered a factor of good prognosis in patients with MM [41,55], our data did not show significant differences in OS and PFS among patients $\mathrm{CD}_{117^{+}}$and $\mathrm{CD} 117$, when it was evaluated as an isolated marker. However, in positive MRD patients its expression was significantly associated to higher probability of OS. Considering these data, it is possible that CD117 expression may identify a subgroup of patients with a better prognosis within a group with a worse prognosis.

In our experience, the expression of 2-microglobulin in the membrane of PCs was not a biomarker to predict the outcome in patients with MM.

According to our results, the effect of GPM and PPM on the survival of MM patients actually demonstrated that the score $\geq 3 \mathrm{GPM}$ can be used as an indicator of better outcome, while a score $\geq 3 \mathrm{PPM}$ can be considered a risk factor for lower survival.

We can consider that there is an inverse correlation between higher levels of MRD and lower number of GPM. On the other hand, there is a direct correlation considering positive MRD and PPM.

Although there was a trend for longer OS and PFS in patients with $\geq 3 \mathrm{GPM}$, the number of GPM had no significance in survival regardless of MRD status. Future studies including more patients and longer follow-up may better elucidate this fact.

In addition, we were able to identify a subgroup of patients with negative MRD with better outcomes. These patients had lower numbers of PPM on clonal PCs and had significantly longer PFS. These associations were not clear in the positive MRD group, with MRD prevailing as the most important prognostic factor.

Although the status of MDR is unquestionably an important predictor of relapse in MM patients, these biomarkers data are relevant and can be included in their follow-up assessments. For example, these markers can be useful to define the frequency for evaluating MRD, according to the profile of higher or lower risk of relapse observed in each patient.

\section{Conclusion}

This retrospective study provided a detailed analysis of the role of immunophenotypic markers in Multiple Myeloma patients, revealing the expressions and number of markers with prognostic relevance. We identified subgroups of patients with better prognosis within the group with undetectable MRD up to the level $<10^{-4}$ to $10^{-6}$, according to the expressions of the immunophenotypic markers. However, the persistence of positive pre-ASCT MRD remains the most important predictive factors for relapse. The recognition of immunophenotypic biomarkers can identify MM patients with a higher risk of recurrence, and these markers can potentially be used as an auxiliary tool in their monitoring.

\section{References}

1. Cowan AJ, Allen C, Barac A, Basaleem H, Bensenor I, Curado MP, et al. Global burden of multiple myeloma: A systematic analysis for the global burden of disease study 2016. JAMA Oncol. 2018; 4: 1221-1227.

2. Bray F, Ferlay J, Soerjomataram I, Siegel RL, Torre LA, Jemal A. Global cancer statistics 2018: GLOBOCAN estimates of incidence and mortality worldwide for 36 cancers in 185 countries. CA Cancer J Clin. 2018; 68: 394-424.

3. Rajkumar SV, Kumar S. Plasma Cell Neoplasms. In: DeVita. VT, Lawrence TS, Rosenberg SA, editors. Cancer Principles and Practice of Oncology 11th ed. Philadelphia: Wolters Kluwer. 2019.

4. Kyle RA, Rajkumar SV. Treatment of Multiple Myeloma: A Comprehensive Review. Clin Lymphoma Myeloma. 2009; 9: 278-288.

5. Rajkumar SV, Kumar S. Multiple myeloma current treatment algorithms. Blood Cancer J. 2020; 10: 94.

6. Al Hamed R, Bazarbachi AH, Malard F, Harousseau JL, Mohty M. Current status of autologous stem cell transplantation for multiple myeloma. Blood Cancer J. 2019; 9: 44.

7. Ntanasis-Stathopoulos I, Gavriatopoulou M, Kastritis E, Terpos E, Dimopoulos MA. Multiple myeloma: Role of autologous transplantation. Cancer Treat Rev. 2020; 82: 101929.

8. Paiva B, Van Dongen JJM, Orfao A. New criteria for response assessment: Role of minimal residual disease in multiple myeloma. Blood. 2015; 125: 3059-3068.

9. Mateo G, Montalbán MA, Vidriales MB, Lahuerta JJ, Mateos M V, Gutiérrez $\mathrm{N}$, et al. Prognostic value of immunophenotyping in multiple myeloma: A study by the PETHEMA/GEM cooperative study groups on patients uniformly treated with high-dose therapy. J Clin Oncol. 2008; 26: 2737-2744. 
10. Paiva B, Almeida J, Pérez-Andrés M, Mateo G, López A, Rasillo A, et al. Utility of flow cytometry immunophenotyping in multiple myeloma and other clonal plasma cell-related disorders. Cytom Part B-Clin Cytom. 2010; 78: 239-252.

11. Paiva B, Gutiérrez NC, Chen X, Vídriales MB, Montalbán MÁ, Rosiñol L, et al. Clinical significance of CD81 expression by clonal plasma cells in high-risk smoldering and symptomatic multiple myeloma patients. Leukemia. 2012; 26 1862-1869.

12. Flores-Montero J, de Tute R, Paiva B, Perez JJ, Böttcher S, Wind H, et al. Immunophenotype of normal vs. myeloma plasma cells: Toward antibody panel specifications for MRD detection in multiple myeloma. Cytom Part B Clin Cytom. 2016; 90: 61-72.

13. Johnsen HE, Bøgsted M, Klausen TW, Gimsing P, Schmitz A, Kjærsgaard $\mathrm{E}$, et al. Multiparametric flow cytometry profiling of neoplastic plasma cells in multiple myeloma. Cytom Part B - Clin Cytom. 2010; 78: 338-347.

14. Kumar S, Paiva B, Anderson KC, Durie B, Landgren O, Moreau P, et al. International Myeloma Working Group consensus criteria for response and minimal residual disease assessment in multiple myeloma. Lancet Oncol. 2016; 17: e328-e346.

15. Rawstron AC, Child JA, De Tute RM, Davies FE, Gregory WM, Bell SE, et al. Minimal residual disease assessed by multiparameter flow cytometry in multiple myeloma: Impact on outcome in the Medical Research Council Myeloma IX study. J Clin Oncol. 2013; 31: 2540-2547.

16. Rawstron AC, Orfao A, Beksac M, Bezdickova L, Brooimans RA, Bumbea $\mathrm{H}$, et al. Report of the European Myeloma Network on multiparametric flow cytometry in multiple myeloma and related disorders. Haematologica. 2008; 93: 431-438

17. Paiva B, Gutie NC, Rosin L. High-risk cytogenetics and persistent minima residual disease by multiparameter flow cytometry predict unsustained complete response after autologous stem cell transplantation in multiple myeloma. Blood. 2012; 119: 687-692.

18. Fulciniti M, Munshi NC, Martinez-Lopez J. Deep Response in Multiple Myeloma: A Critical Review. Biomed Res Int. 2015

19. Rawstron AC, Gregory WM, De Tute RM, Davies FE, Bell SE, Drayson MT, et al. Minimal residual disease in myeloma by flow cytometry: Independen prediction of survival benefit per log reduction. Blood. 2015; 125: 1932-1935.

20. Paiva B, Vidriales MB, Cerveró J, Mateo G, Pérez JJ, Montalbán MA, et al. Multiparameter flow cytometric remission is the most relevant prognostic factor for multiple myeloma patients who undergo autologous stem cell transplantation. Blood. 2008; 112: 4017-4023.

21. FDA. Hematologic Malignancies: Regulatory Considerations for Use of Minimal Residual Disease in Development of Drug and Biological Products for Treatment Guidance for Industry. 2018.

22. Kumar S, Kimlinger T, Morice W. Immunophenotyping in multiple myeloma and related plasma cell disorders. Best Pract Res Clin Haematol. 2010; 23 . 433-451.

23. Mathis S, Chapuis N, Borgeot $\mathrm{J}$, Maynadié $\mathrm{M}$, Fontenay $\mathrm{M}$, Béné $\mathrm{MC}$, et al. Comparison of cross-platform flow cytometry minimal residual disease evaluation in multiple myeloma using a common antibody combination and analysis strategy. Cytom Part B - Clin Cytom. 2015; 88: 101-109.

24. Anderson KC, Auclair D, Kelloff GJ, Sigman CC, Avet-Loiseau H, Farrell AT, et al. The role of minimal residual disease testing in myeloma treatment selection and drug development: Current value and future applications. Clin Cancer Res. 2017; 23: 3980-3993.

25. Meddour Y, Rahali MC, Belakehal SE, Benfenatki N, Ardjoune FZ, Chaib S, et al. Plasma Cell Immunophenotyping Improve Prognostic Stratification of Multiple Myeloma Patients. Int J Cancer Manag. 2018; 11: 1-9.

26. Maurer D, Holter W, Majdic O, Fischer GF, Knapp W. CD27 expression by a distinct subpopulation of human B lymphocytes. Eur J Immunol. 1990; 20 2679-2684.

27. Raja KRM, Kovarova L, Hajek R. Review of phenotypic markers used in flow cytometric analysis of MGUS and MM, and applicability of flow cytometry in other plasma cell disorders. Br J Haematol. 2010; 149: 334-351.

28. Moreau P, Robillard N, Jégo G, Pellat C, Gouill S Le, Thoumi S, et al. Lack of CD27 in myeloma delineates different presentation and outcome. $\mathrm{Br} \mathrm{J}$ Haematol. 2006; 132: 168-170.

29. Arana P, Paiva B, Cedena MT, Puig N, Cordon L, Vidriales MB, et al. Prognostic value of antigen expression in multiple myeloma: A PETHEMA/ GEM study on 1265 patients enrolled in four consecutive clinical trials. Leukemia. 2018; 32: 971-978.

30. Morgan TK, Zhao S, Chang KL, Haddix TL, Domanay E, Cornbleet PJ, et al. Low CD27 expression in plasma cell dyscrasias correlates with high-risk disease: An immunohistochemical analysis. Am J Clin Pathol. 2006; 126: 545-551.

31. Guikema JEJ, Hovenga S, Vellenga E, Condradie JJ, Abdulahad WH, Bekkema R, et al. CD27 is heterogeneously expressed in multiple myeloma: Low CD27 expression in patients with high-risk disease. Br J Haematol. 2003; 121: $36-43$.

32. Murray ME, Gavile CM, Nair JR, Koorella C, Carlson LM, Buac D, et al. CD28-mediated pro-survival signaling induces chemotherapeutic resistance in multiple myeloma. Blood. 2014; 123: 3770-3779.

33. Bahlis NJ, King AM, Kolonias D, Carlson LM, Liu HY, Mohamad A, et al. CD28-mediated regulation of multiple myeloma cell proliferation and survival. 2007; 109: 5002-5010

34. Hermiston ML, Xu Z, Weiss A. CD45: A Critical Regulator of Signaling Thresholds in Immune Cells. Annu Rev Immunol. 2003; 21: 107-137.

35. Kumar S, Rajkumar SV, Kimlinger T, Greipp PR, Witzig TE. CD45 expression by bone marrow plasma cells in multiple myeloma: clinical and biological correlations. Leukemia. 2005; 19: 1466-1470.

36. Moreau P, Robillard N, Avet-Loiseau H, Pineau D, Morineau N, Milpied N et al. Patients with CD45 negative multiple myeloma receiving high-dose therapy have a shorter survival than those with CD45 positive multiple myeloma. Haematologica. 2004; 89: 547-551.

37. Mathew P, Ahmann GJ, Witzig TE, Roche PC, Kyle RA, Greipp PR Clinicopathological correlates of CD56 expression in multiple myeloma: a unique entity?. Br J Haematol. 1995; 90: 459-461.

38. Sahara N, Takeshita A, Shigeno K, Fujisawa S, Takeshita K, Naito K, et al Clinicopathological and prognostic characteristics of CD56-negative multiple myeloma. Br J Haematol. 2002; 1: 882-885.

39. Hundemer M, Klein U, Hose D, Raab MS, Cremer FW, Jauch A, et al. Lack of CD56 expression on myeloma cells is not a marker for poor prognosis in patients treated by high-dose chemotherapy and is associated with translocation (11;14). Bone Marrow Transplant. 2007; 40: 1033-1037.

40. Skerget M, Skopec B, Zadnik V, Zontar D, Podgornik H, Rebersek K, et al. CD56 Expression Is an Important Prognostic Factor in Multiple Myeloma even with Bortezomib Induction. Acta Haematol. 2018; 139: 228-234.

41. Pan Y, Wang H, Tao Q, Zhang C, Yang D, Qin H, et al. Absence of both CD56 and CD117 expression on malignant plasma cells is related with a poor prognosis in patients with newly diagnosed multiple myeloma. Leuk Res. 2016; 40: 77-82.

42. Perez-Andres $M$, Almeida J, Martin-Ayuso $M$, De Las Heras N, Moro MJ, Martin-Nuñez G, et al. Soluble and membrane levels of molecules involved in the interaction between clonal plasma cells and the immunological microenvironment in multiple myeloma and their association with the characteristics of the disease. Int J Cancer. 2009; 124: 367-375.

43. Branagan A, Lei M, Lou U, Raje N. Current Treatment Strategies for Multiple Myeloma. Clin Rev. 2020; 16: 5-15.

44. Kalina T, Flores-Montero J, Van Der Velden VHJ, Martin-Ayuso M, Böttcher $\mathrm{S}$, Ritgen $\mathrm{M}$, et al. EuroFlow standardization of flow cytometer instrument settings and immunophenotyping protocols. Leukemia. 2012; 26: 1986-2010.

45. Van Dongen JJM, Lhermitte L, Böttcher S, Almeida J, Van Der Velden $\mathrm{VHJ}$, Flores-Montero J, et al. EuroFlow antibody panels for standardized n-dimensional flow cytometric immunophenotyping of normal, reactive and malignant leukocytes. Leukemia. 2012; 26: 1908-1975. 
46. Flores-Montero J, Sanoja-Flores L, Paiva B, Puig N, García-Sánchez O, Böttcher S, et al. Next Generation Flow for highly sensitive and standardized detection of minimal residual disease in multiple myeloma. Leukemia. 2017; 31: 2094-2103.

47. R Core Team. R: A Language and Environment for Statistical Computing Vienna, Austria: R Foundation for Statistical Computing; 2018.

48. Landgren O, Devlin S, Boulad M, Mailankody S. Role of MRD status in relation to clinical outcomes in newly diagnosed multiple myeloma patients: A meta-analysis. Bone Marrow Transplant. 2016; 51: 1565-1568.

49. Munshi NC, Avet-Loiseau H, Rawstron AC, Owen RG, Child JA, Thakurta A, et al. Association of minimal residual disease with superior survival outcomes in patients with multiple myeloma: A meta-analysis. JAMA Oncol. 2017; 3 28-35.

50. Paiva B, Puig N, Cedena MT, Jong BG De, Ruiz Y, Rapado I. Differentiation stage of myeloma plasma cells : biological and clinical significance. Leukemia. 2017; 31: 382-392.

51. Pellat-Deceunynck C, Bataille R. Normal and malignant human plasma cells: Proliferation, differentiation, and expansions in relation to CD45 expression. Blood Cells, Mol Dis. 2004; 32: 293-301.
52. Pellat-Deceunynck C, Barillé S, Jego G, Puthier D, Robillard N, Pineau D, et al. The absence of CD56 (NCAM) on malignant plasma cells is a hallmark of plasma cell leukemia and of a special subset of multiple myeloma. Leukemia. 1998; 12: 1977-1982.

53. Khallaf S, Yousof E, Ahmed E, Mansor S, Mohamed H, Elgammal S, et al Prognostic Value of CD56 Expression in Multiple Myeloma. Res Oncol. 2020; $0: 1-5$

54. Ely SA, Knowles DM. Expression of CD56/neural cell adhesion molecule correlates with the presence of lytic bone lesions in multiple myeloma and distinguishes myeloma from monoclonal gammopathy of undetermined significance and lymphomas with plasmacytoid differentiation. Am J Pathol. 2002; 160: 1293-1299.

55. Schmidt-Hieber M, Pérez-Andrés M, Paiva B, Flores-Montero J, Perez JJ, Gutierrez NC, et al. CD117 expression in gammopathies is associated with an altered maturation of the myeloid and lymphoid hematopoietic cell compartments and favorable disease features. Haematologica. 2011; 96: 328-332. 